(c) 2010 IEEE. Personal use of this material is permitted. Permission from IEEE must be obtained for all other uses, in any current or future media, including reprinting/republishing this material for advertising or promotional purposes, creating new collective works, for resale or redistribution to servers or lists, or reuse of any copyrighted component of this work in other works. 


\section{Applications of Wireless Sensor Networks in the Oil, Gas and Resources Industries}

\author{
Mohammad reza Akhondi, Alex \\ Talevski \\ DEBII \\ Curtin University of Technology, \\ Perth, Western Australia \\ M.Akhondi@postgrad.curtin.edu.au \\ A.Talevski@curtin.edu.au
}

\author{
Simon Carlsen \\ Statoil ASA, Trondheim, \\ Norway \\ SCAR@StatoilHydro.com
}

\author{
Stig Petersen \\ SINTEF ICT, Trondheim, \\ Norway \\ stig.petersen@sintef.no
}

\begin{abstract}
The paper provides a study on the use of Wireless Sensor Networks (WSNs) in refineries, petrochemicals, underwater development facilities, and oil and gas platforms. The work focuses on networks that monitor the production process, to either prevent or detect health and safety issues or to enhance production. WSN applications offer great opportunities for production optimization where the use of wired counterparts may prove to be prohibitive. They can be used to remotely monitor pipelines, natural gas leaks, corrosion, H2S, equipment condition, and real-time reservoir status. Data gathered by such devices enables new insights into plant operation and innovative solutions that aids the oil, gas and resources industries in improving platform safety, optimizing operations, preventing problems, tolerating errors, and reducing operating costs. In this paper, we survey a number of WSN applications in oil, gas and resources industry operations.
\end{abstract}

Keywords: Wireless Sensor Network, Production Optimization, Reservoir Monitoring, Condition Monitoring and Maintenance, Health and Safety.

\section{INTRODUCTION}

The oil and gas industry includes processes for exploration, extraction, refining, transporting, and marketing petroleum products. The largest volume products of the industry are fuel, oil and gasoline (petrol). Petroleum is also the raw material for many chemical products, including pharmaceuticals, solvents, fertilizers, pesticides, and plastics. As the demand for fossil fuels continues to grow, oil and gas companies will have to develop new technologies and improve operations in order to increase productivity and expand on their current abilities.

Furthermore, environmental regulations are constantly changing and becoming stricter day by day. In 2008, The Office of the Comptroller of the Currency (OCC) approved a set of rules on the management of surface waste from oil and gas operations that force companies to haul highly contaminated soil and water to permanent disposal sites rather than spread it back over the land after closing a well. The OCC also approved stricter penalties to enforce industry compliance with environmental standards [1]. Oil and gas companies must develop new methods to abide by new regulations and, reduce accidents and emissions without impacting production. Due to the evolution of digital technologies and wireless communications, wireless sensor networks can be quickly organized and continually adapted to monitor and control environmental conditions and machinery in response to business drivers and requirements.

\section{WIRELESS SENSOR NETWORKS (WSNS)}

According to a recent report [2], WSNs will be the only feasible sensing solution for the Oil \& Gas industry, which is projected to spend \$200M on the technology between 2009 and 2012. WSN technology is ideal for this industry for the following reasons;

- The numerous remote and hazardous locations

- The difficulty and cost in introducing new wired devices near pipelines

- The requirement for temporary sensor installation

- The requirement for sensor redundancy

- The evolution of new control solutions that require further sensors

- The demand for continuous production optimization and growth

- The demand for improved safety

Some specific highlights of the report are [2]:

- One quarter of all industrial wireless sensors deployed in 2009 will be for oil and gas applications

- Refineries will be the largest and first segment to take off

- Most research and development is focused on wireless condition based monitoring (CBM) for preventive maintenance.

Recent advances in wireless technology have enabled the development of low-cost wireless solutions capable of robust and reliable communication. International standards such as the IEEE $802.11 \mathrm{a} / \mathrm{b} / \mathrm{g}$ [3][4][5][6] for wireless local area networks (often referred to as Wi-Fi) and the IEEE 802.15.4 [7] for low-rate wireless Personal Area Networks (PANs), as well as numerous RFID (Radio-Frequency Identification) 
specifications, have enabled applications such as wireless networking, sensing, monitoring, control, and asset tracking solutions.

Wireless technology has the potential to be beneficial in many regards [8]. Eliminating the need for cables can contribute to reduced installation and operating costs, it enables installations in remote areas, and it allows for costefficient, temporary and mobile systems. In addition, wireless technology will enable a new range of applications; particularly in the area of health, safety and environment (HSE).

Networks may be composed of many different types of sensors such as seismic, thermal, visual, acoustic, radar, magnetic, infrared, and many others which are able to monitor a wide variety of ambient conditions such as the following [9][10]: Environmental weather conditions, Pressure, Temperature (Including infrared maps), Gas, Fire, Sound, Humidity, Vibration, Electrical characteristics, Mechanical stress, Soil makeup, Object movement, Presence or absence of objects, Speed, direction, and size of an object, Location etc.

Furthermore, the concept of micro-sensing and wireless connection of these nodes promises many new application areas [11]

Recently, wireless sensor networks have found their way into a wide variety of applications and systems with varying requirements and characteristics [12]. Some sample applications are: Bird observation, Glacier monitoring, Cattle herding, Bathymetry, Ocean water monitoring, Grape monitoring, Cold chain management, Avalanche rescue, vital sign monitoring, Power monitoring, Parts assembly, Object tracking, and many others [12]

\section{WSN STANDARDS}

Specifications for international standards for wireless sensor networks, such as ZigBee PRO [13], Wireless HART [14] and ISA100.11a [15], use stacks to provide a layered and abstract description of the network protocol design [16].

Each layer in the stack is a collection of related functions, and a layer is responsible for providing services to the layer above it, while it receives services from the layer below it.

- The Physical Layer (PHY) - controls the RF transceiver, performs frequency and channel selection, handles energy and signal management functions, and provides means for transmitting raw bits (not packets).

- The Medium Access Control Layer (MAC) handles access to the physical radio channel, is responsible for radio synchronization, and provides a reliable link between two peer MAC entities.

- The Network Layer - is responsible for joining and leaving a network, it provides end-to-end packet delivery from source to destination, and it discovers and maintains routing tables.
- The Application Layer - provides services to user defined application processes (not necessarily to the end-user), it handles fragmentation and reassembly of data packets, and it defines the role of the device within the network (coordinator, router or enddevice).

Even though, through its layers, a stack defines and describes the functionality and operation of a wireless device for a given standard, it does not provide any guidelines on how to develop and implement the embedded software that is needed in order to realize the protocols described in the standard specifications. As a result, creating software stacks for international standards has become a major challenge. Not only will the software stack have to implement complex network protocols with real-time requirements in an embedded environment with scarce resources, it also has to fulfill the requirement of guaranteed interoperability between the devices and the software stacks from different vendors.

Energy efficiency is a key consideration in the development of every wireless device and network component, with the goal of maximizing the period before maintenance (battery replacement) is required. Moreover, wireless monitoring and control applications for oil, gas and resources industries require longer battery life and less complexity than those available from existing wireless standards. An IEEE 802.15.4 task group is investigating a low data rate solution with multi-month to multi-year battery life and low complexity. A highly integrated single-chip approach is the preferred solution of semiconductor manufacturers developing IEEE 802.15.4 compliant transceivers. The IEEE standard at the PHY is the significant factor in determining the radio frequency (RF) architecture, and topology of transceivers [17].

\section{A. Device Performance}

To provide for low-cost implementation options, the physical devices are distinguished based on the IEEE 802.15.4 definition of reduced function device (RFD) and full function device (FFD). An IEEE 802.15.4 network requires at least one FFD to act as a network coordinator. An RFD sees implementation with minimum RAM and ROM resources as a simple send or receive node in a larger network. With a reduced stack size, it needs less memory, and thus a less expensive IC. RFDs are generally battery powered. RFDs can search for available networks, transfer data from its application as necessary, determine whether data is pending, request data from the network coordinator, and sleep for extended periods of time to reduce battery consumption. RFDs can only talk to an FFD, a device with sufficient system resources for network routing. The FFD can serve as a network coordinator, a link coordinator, or as just another communications device. Any FFD can talk to other FFD and RFDs. FFDs discover other FFDs and RFDs to establish communications, and they're typically line powered [17].

\section{B. Network Performance}

Self-forming and self-healing mesh network architectures permit data and control messages to pass from one node to other node via multiple paths. This extends the range of the 
network and improves data reliability. You could use the peer-to-peer capability to build large, geographically dispersed networks where smaller networks link together to form a cluster-tree network [17].

Networks may consist of multiple traffic types with their own unique characteristics, including periodic data, intermittent data, and repetitive low latency data. The characteristics of each are as follows:

- Periodic data - is usually defined by the application such as a wireless sensor or meter. You would typically handle data using a beaconing system where the sensor wakes up and checks for the beacon, exchanges data, and goes to sleep.

- Intermittent data - is either application- or external stimulus-defined, such as a wireless light switch. You can handle data in a beaconless system or a disconnected operation.

- Repetitive low latency data - uses time slot allocations, such as a security system. These applications may use the guaranteed time slot (GTS) capability. GTS is a method of QoS that allows each device a specific duration of time as defined by the PAN coordinator in the Superframe to do whatever it requires without contention or latency

Networks are primarily intended for low duty cycle sensor networks $(<1 \%)$. They may recognize new network nodes and associate those in about $30 \mathrm{~ms}$. Waking up a sleeping node may take about $15 \mathrm{~ms}$, as does accessing a channel and transmitting data. Applications benefit from the ability to quickly attach information, detach, and go to deep sleep, which results in low power consumption and extended battery life [17].

Many researchers are currently engaged in developing the technologies needed for different layers of the sensor networks protocol stack to be more applicable for monitoring and control in the oil and gas industries.

The most challenging part of designing protocol stack is managing the power used by the devices. The major source of power consumption in a wireless sensor is mainly the communication layer RF transceiver. To conserve power, it is therefore imperative to be able to shut down the transceiver when it is not in use. The ability to shut down the transceiver can also be beneficial for the power consumption of the processing unit, as in many cases, the microprocessor can enter low-power sleep modes when there is no need for communication. However, power consumption when starting the transceiver is much higher than when it is in use, the average power consumption might actually be larger by turning the unit on and off, instead of leaving it on all the time [18].

A common method to achieve reduced power consumption is to synchronize the network with a time-division multiple access (TDMA) algorithm. All network communication is divided into distinct timeslots of equal length. A timeslot is usually so long as to allow enough time for a device to transmit one packet and wait for the reception of an acknowledgement from the recipient. Each communication link between two devices in the network is given two unique timeslots (the two devices alternate between the roles of transmitter and receiver in the two timeslots), thus enabling contention-free communication throughout the network, and enabling each device to enter a low power sleep mode with its transceiver turned off in all the timeslots not reserved for its own links. A weakness with this form of division of network traffic is its inability to scale in an efficient manner. To combat this, hybrid TDMA algorithms can be used, where a certain number of timeslots in the frame is not dedicated to a given communication link, but open to free contention between the devices in the network [19]. In Time Division Multiple Access (TDMA), the RF link is divided on a time axis, with each node being given a predetermined time slot it can use for communication. This decreases the sweep rate, but a major advantage is that TDMA can be implemented in software. All nodes require accurate, synchronized clocks for TDMA [20].

Wireless devices usually have very limited resources due to strict low-power requirements. However, they are still required to execute software implementations of complex networking algorithms with real-time requirements. They have to be capable of self-healing and self-configuration in order to provide a robust and reliable multi hop network for rough RF environments. This can be achieved by the use of dynamic routing protocols, where each wireless sensor has to store and constantly update neighbor information, as well as handling network connection requests from other wireless sensors. Importantly, they must provide self configurable, dynamic and adaptive application services [19].

\section{OIL AND GAS REQUIREMENTS}

The technical requirements for the deployment of wireless technology in the oil, gas and resources industries have been identified in [8]. As wireless technologies have developed, we are currently seeing the use of wireless sensors being installed on older end-of-lifecycle platforms in order to gain new insights and optimize production [21]. There are several challenges related to the introduction of such wireless devices in the oil, gas and resources industries. Such wireless devices must have the following properties;

- Restricted size, shape, construction and certification.

- Make do with limited processing power, memory, storage, battery consumption and screen real-estate.

- Self contained. When possible, devices should generate their own power or, contain battery packs with extended battery life of many years to reduce maintenance requirements.

- Operate in a difficult wireless environment both in terms of radio noise and obstructions but also where certain restrictions on such radio devices are present (such as flammable areas).

- Operate in hostile areas where environmental and platform conditions may be very harsh. 
- Embedded platforms. Implement complex network algorithms with real-time requirements and adaptive routing protocols.

- Contribute in a simplified ad-hoc and multi-hop network.

- Seamlessly integrate with existing IT solutions.

- Self re-configurable, dynamic and adaptive.

- Provide services within a dynamically changing system environment.

- Exhibit fault tolerance and recovery (self-healing, robust and reliable).

- Based on open, international standards.

- Operate in the unlicensed portions of the frequency spectrum.

- Low maintenance.

- Secure operation and transmission.

- Clearly defined operational reliability and availability of the wireless network within the operational environment.

The studies performed in [22] show that the required changes in factory and plant work processes might be the largest hindrance for the introduction of wireless technology in the oil, gas and resources industries. It has been noted that problems are typically experienced when the human factors are ignored in the adoption of new technology.

\section{APPLICATIONS IN THE OIL AND GAS INDUSTRY}

An accelerating energy crisis in the oil and gas industry is driving development and investment in wireless sensor network (WSN) technologies. WSNs are a key investment area across the oil and gas supply chain including refineries/petrochemical plants, pipelines, exploration \& production, and transportation. By providing secure and reliable two-way wireless communications, WSN enables automation and control solutions that are not feasible with wired systems. This is a multidisciplinary research area requires close collaboration between users, application domain experts, hardware designers, and software developers in order to implement efficient systems.

\section{A. Remote Monitoring}

The Wireless Sensor Network solution provides remote monitoring capabilities for oil and gas companies to meet this new technology, regulatory and production demands. Example Oil \& Gas Remote Monitoring applications using the solution include:

- Pipeline Integrity Monitoring

- Tank Level Monitoring

- Equipment Condition Based Monitoring (CBM)

- Pipeline Pressure Relief Valve Monitoring (PRV)

- Refineries Pressure Relief Valve Monitoring (PRV)

- Wellhead Automation and Monitoring

Offshore (marine environments) are particularly corrosive and require intensive maintenance. Remote Monitoring solutions for oil and gas applications can greatly reduce, and in some cases, eliminate maintenance costs. Due to the rate of production and the cost of the product, these platforms require continuous operation for maximum productivity. Real-time data acquisition and monitoring cuts the cost of complying with regulations, reducing or eliminating down times for maintenance or unplanned machinery failure and allows for energy management and savings by giving the operator a constant view of the state of all aspects of the platform.

WSN technologies enable better, faster and more realtime communications in order to improve productivity, refinery processes, security and regulations compliance. WSN systems provide simpler access to real-time data and insight into field operations for streamlined operations.

The efficient and effective movement of natural gas from producing regions to consumption regions requires extensive and elaborate transportation systems. In many instances, natural gas produced from a particular well will have to travel a great distance to reach its point of use. The transportation system for natural gas consists of a complex network of pipelines, designed to quickly and efficiently transport natural gas from its origin, to areas of high natural gas demand [12]. Hence, natural gas detecting equipment is periodically used by pipeline personnel on the surface to check for leaks. This is especially important in areas where the natural gas is not odorized.

In the USA, the underground piping includes approximately 400,000 miles of transmission pipelines and 1.4 million miles of distribution piping, while above-ground piping is located mainly at about 750 gas processing plants and some 3000 compressor stations. These pipelines are often disrupted by leaks. Regulatory pressure is increasing to inspect transmission lines more frequently. Remote gas leak survey is a proactive way to prevent unnecessary loss of human life resulting from natural gas leaks. Since the existing active detection system is too expensive and a passive detection system is less reliable, a WSN spectroscopic system that utilizes the sunlight to detect the methane within $850-950 \mathrm{~nm}$ wavelength range can be used. Being able to detect methane within this range is significant.

\section{B. Condition Monitoring and Maintenance}

Fault diagnosis can be divided into component fault diagnosis and system fault diagnosis. The aim of fault diagnosis is the estimation of the component status through sensor measurements and careful monitoring of system components. Equipment diagnosis tries to determine the cause of a failure of a component whereas system diagnosis is performed on a system of composite components. Using sensor measurement, preventive and predictive maintenance can be performed and post-fault diagnosis is improved. Predictive maintenance methodologies require that components are monitored in real time. Conventional techniques apply simplified linear pattern matching methods such as linear level threshold algorithms. Sensors may detect vibration, temperature heat, dissolved gas, electromagnetic properties, power consumption, performance etc. When combined with other sensor outputs, these continuous signals represent significant information about the condition of a component. The sensor signals are processed and compared 
and a component state can be determined. However, when such a setup exists, such systems can detect and even predict potential upcoming component faults. This may limit downtime, repair costs, damage and potential danger. Component fault diagnosis can be performed off-line without direct coupling to real-time data and processing. Fault diagnosis is a pattern recognition and classification task which has been investigated with the use of neural networks [23].

Corrosion monitoring can be used as a diagnostic tool to track plant condition. It confirms expected conditions and draws attention to anything that is out of specification. It provides a management tool to assist;

- Asset integrity management

- Performance effectiveness and differentiation of competitive corrosion inhibitors

- Evaluate and select different materials and metallurgy to ensure materials last as long as possible

- Optimize production

- Define operational constraints in terms of duration, speed, intensity and durability

- Reduced operation costs and economics

- Optimize operations and plant condition through effective and efficient maintenance

- Improved safety and reduced risk

- Determine operational variations which cause system degradation and failure aid decision making

\section{H2S Monitoring}

During oil exploration and refinery processes, many types of toxic gases are produced as product or by-product [24]. These include Ammonia (NH3), Hydrogen Sulphide (H2S), and Sulphur Dioxide (SO2). Among which H2S is a useful and common by-product, but also a cause of health and corrosion problems. It is used to recover sulphur, which is commonly used for manufacturing sulphuric acid, medicine, cosmetics, fertilizers and rubber products. During oil extraction and refinery processes, H2S is carefully transported in pipelines to workshops where further processing takes place. The main concern at this stage is the potential of leaks that can have a severe impact on human beings as well as the environment. Leakages also mean putting a pipeline out of service for repair. The cause of leakages can be excessive deformations caused by earthquakes, corrosion, wear and tear, material flaws or even intentional damage [24].

Hydrogen sulphide is an extremely toxic, colorless, flammable gas that is heavier than air and soluble in water. It has a rotten egg odor, which is discernible at concentrations well below its very low exposure limit. Exposure to low levels of hydrogen sulphide will cause irritation, dizziness and headaches, while exposure to levels in excess of the prescribed limits will cause nervous system depression and, eventually, death [25]. Besides the harm to human beings, $\mathrm{H} 2 \mathrm{~S}$ has also a negative impact on the ecological system. For instance, $\mathrm{H} 2 \mathrm{~S}$ in water may change the $\mathrm{PH}$ value, which would eventually result in an ecological imbalance between the microbes and aquatic species in that habitat. Subsequently, various parties are interested in monitoring $\mathrm{H} 2 \mathrm{~S}$ release during oil exploration and refinery using WSNs [24].

Pipeline ownership entails considerable management challenges because of the long length, high value, high risk and often difficult access conditions. A pipeline inspection task involves both a stationary sensing system and portable sensors carried by maintenance workers. A stationary sensing system consists of sensors, power and signal cables and a control station in which the sensed data are processed. Because of the initial installation as well as maintenance cost, the deployed sensing system may not adequately cover the sensing field. As a result, each employee is required to carry with him a portable sensing device for safety reasons. A WSN approach here may give a cross section of any potential leaks for broader analysis. Existing sensing systems consider reports from individual sensors independently; they do not correlate reports from spatially distributed sensors in order to determine the nature of a leakage report. For example, correlation of sensed data can be helpful not only to detect and localize a leakage, but also to determine whether a leakage report made by multiple sensors is a result of actual leakages or diffusion of the gas from a single source [24].

\section{Production Performance}

Given an appropriate amount of data from various performance aspects of an oil and gas functions as facilitated by the flexibility of WSNs, an unsupervised Self Organising Map (SOM) can prioritize key sensor values and classify operational performance. It can show when a plant is operating normally and abnormally. Such networks are often used in combination with supervised approaches where the unsupervised networks perform data pre-processing and the supervised networks estimate the associated parameters.

The Hopfield network can be used to analyze optimal load flow, unit commitment and economic dispatch [26]. As a result of the Hopfield net's large number of local minima, a local optimum can be found quite quickly. This value can then be used with other optimization techniques for improved accuracy. Such control systems must be operated on-line because of the requirement for rapid reaction to operational conditions and the significant impact that is possible to other interconnected systems. Therefore, control systems are still optimized in the most conservative manner. New control tools need to be extensively tested before they can be integrated into such complex operating environments.

Sensor data can be used to realize certain characteristics and patterns in operation data. Such solutions may enhance operational visualization, foresight, forecasting and maintenance schedules for effective and efficient operation and maintenance. This optimizes plant safety, production, turnarounds, shutdowns and maintenance and improves error tolerance and recovery. 


\section{VI. Well REMOTE Monitoring SyStem}

In this section we are trying to present one case study which is related using wireless sensor networks in the oil and gas industry. So, we will discuss a well remote monitoring system which is very important because of two main reasons. Firstly, safety and secondly cost. In the oil and gas industry, more exploration and production is occurring in areas that were never considered. Exploratory drilling is occurring in backyards, school yards and community parks. In many communities, well heads or pumping units are being installed within or very close to residential communities. It is important that the members of these communities feel safe and not threatened by gas exposure. To protect these areas, remote monitoring systems can be easily deployed while drilling occurs, or placed permanently while the wells are producing oil and gas.

\section{A. System Introduction}

With the help of data monitoring system of oil wells, managerial staff can learn about the production status of oil wells at any time without going outside. The data of oil wells is transmitted every two hours. Once the oil well gets problems, the diagram will display exclamatory mark. The maintenance staff rushes to the field in the shortest time to solve the problem. After employing 3G/GPRS drilling data submit system, through the sensor fixed on the rack, managerial staff can learn about the pressure, voltage, steam temperature of each well, guaranteeing the safety operation of oil wells [27].

\section{B. System Structure}

According to the characteristics of oil wells distribution and the development level of wireless communication technology, the remote monitoring and control system of oil wells introduces CDMA/GPRS or data transceiver communication mode. The system consists of sensor, well head controller SU, CDMA/GPRS Data Transfer Unit (DTU), oil production plant communication equipment, realtime database server, WEB server, monitoring browse terminal, etc. A complete monitoring system of oil field should comprise three parts: local monitoring system of oil field, central acquisition server of information center and local area network (LAN) of plant.

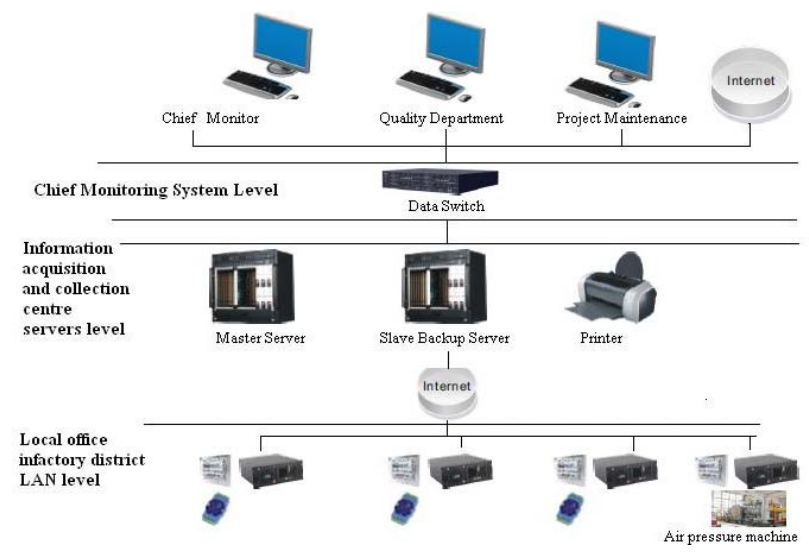

Figure 1: System Structure

\section{The Principles of Local Monitoring System}

The local monitor of oil field consists of data acquisition module, 3G/GPRS communication module, Remote Terminal Unit (RTU), field data acquisition computer and local central server. In SCADA systems, an RTU is a device installed at a remote location that collects data, codes the data into a format that is transmittable and transmits the data back to a central station, or master. An RTU also collects information from the master device and implements processes that are directed by the master. RTUs are equipped with input channels for sensing or metering, output channels for control, indication or alarms and a communications port. The transceiver of monitoring center receives the signals from the air delivered by oil field RTU, and then amplifies and demodulates RF signals, restores the signals into data transfer signals and sends them to central industrial control computer to calculate, real-time monitors oil well status. When something appears abnormal, the central Industrial PC sends out commands to the RTU of oil field to stop motor and wait for maintenance. The whole system runs automatically and can be monitored without human interference [27].

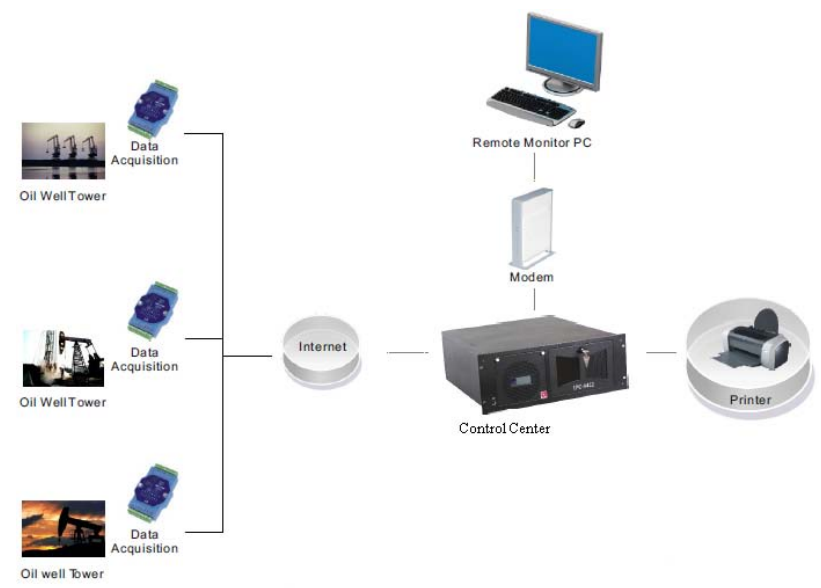

Figure 2: The principles of Local Monitoring System

\section{System Functions}

The sensors for detecting oil well operation mainly includes temperature sensor, pressure sensor, motor current and voltage sensor, the load and displacement of oil pump sensor, transformer. They convert the operating status of oil wells into corresponding voltage or current and deliver them to the RTU of well head. And then it connects to the communication equipment of oil production plant through CDMA/GPRS network or data transfer broadcasting station. Data communication equipment collects the controller data of well head by means of polling method. After being analyzed and processed, the data goes to the real-time database of the oil plant. And different departments of the oil plant can access the real-time database according to their rights by means of $\mathrm{C} / \mathrm{S}$ or $\mathrm{B} / \mathrm{S}$ mode. 
1) Monitors the operating status of oil well

a) Monitors the power supply voltage of oil pump

b) Monitors the power supply current of oil pump

c) Monitors motor on/off

d) Monitors oil leaking, oil stolen

2) Real-time alarm the fault of oil well

a) Gives alarm in case of over-voltage or undervoltage

b) Gives alarm in case of over-current

c) Gives alarm in case that oil pump aborts

d) Gives alarm in case of oil leaking, oil stolen

3) Monitors data statistics and prints

a) Computer real-time displays power consumption and faults and prints statistic report

4) Expandability

a) The system leaves enough expansion interfaces for users to expand more features according to oil well condition and requirements [27].

\section{FUTURE WORK}

The most general and versatile deployments of wireless sensing networks demand that batteries be deployed. Future work is being performed on systems that exploit piezoelectric materials to harvest ambient strain energy for energy storage in capacitors and/or rechargeable batteries. By combining smart, energy saving electronics with advanced thin film battery chemistries that permit infinite recharge cycles, these systems could provide a long term, maintenance free, wireless monitoring solution. We also want to improve underwater development and production of oil and gas needs networked sensors and actuators to monitor the production process, to either prevent or detect oil and gas leakage or to enhance the production flow and yield of the wells. Sensors used today are large and expensive, both to manufacture and to deploy sub-sea. We suggest sensor networks created by inexpensive and small wireless sensor nodes in a redundant multi-hop topology. Preliminary designs and results are shown for two such nodes using ultrasound and infrared light as wireless commutation [28].

\section{CONCLUSIONS}

In this paper, a survey of the related WSNs and research areas in the oil, gas and resources industries has been presented. Wireless sensors and devices are able to monitor plant performance and the operational environment of oil, gas and resource production plants. When effectively deployed, well developed sensor solutions promote a safe and healthy workplace and simultaneously give the ability to optimize production, and operation safety. Wireless technologies as such are fit for deployment, and can provide benefits such as improved platform safety, optimized operations, preventing problems, tolerating errors, and reducing operating costs. However, without an appropriate study on how they can be incorporated into existing work processes, these benefits cannot be fully realized. It is expected that wireless technologies will soon enable significant cost effective applications of both a temporary, mobile and permanent nature in remote or hostile areas in the oil, gas and resources industries.

\section{REFERENCES}

[1] Oil and gas industry fights state environmental regulations (cited 200922 August); Available from: $h$ ttp://albuquerque.bizjournals.com/albuquerque/stories /2007/10/22/story4.html .

[2] Wireless Sensor Networks for Oil \& Gas. 2008 (cited 200922 July); Available from: http://www.onworld.com

[3] IEEE Computer Society, "IEEE 802.11-1999: Wireless LAN Medium Access Control (MAC) and Physical Layer (PHY) specifications", Standard, July 1999.

[4] IEEE Computer Society, "IEEE 802.11a-1999: Highspeed Physical Layer in the $5 \mathrm{GHz}$ Band", Standard, Feb. 1999.

[5] IEEE Computer Society, "IEEE 802.11b-1999: Wireless LAN Medium Access Control (MAC) and Physical Layer (PHY) specifications: Higher-Speed Physical Layer Extension in the $2.4 \mathrm{GHz}$ Band", Standard, Oct. 1999.

[6] IEEE Computer Society, "IEEE 802.11g-2003: Wireless LAN Medium Access Control (MAC) and Physical Layer (PHY) specifications: Further Higher Data Rate Extension in the 2.4 GHz Band", Standard, June 2003.

[7] IEEE Computer Society, "IEEE 802.15.4-2006: Wireless Medium Access Control (MAC) and Physical Layer (PHY) Specifications for Low-Rate Wireless Personal Area Networks (LR_WPANs)", Standard, Dec. 2006.

[8] Petersen, S., P. Doyle, S. Vatland, C. S. Aasland, T. M. Andersen, and S. Dag. 2007. Emerging Technologies and Factory Automation, 2007. ETFA. IEEEConference on, Requirements, drivers and analysis of wireless sensor network solutions for the Oil \& Gas industry, pp 102-114.

[9] I.F. Akyildiz, W. Su, Y. Sankarasubramaniam, E. Cayirci, A survey on sensor networks, IEEE Communications Magazine 40 (8) (2002) 104-112.

[10] D. Estrin, R. Govindan, J. Heidemann, S. Kumar, Next century challenges: scalable coordination in sensor networks, ACM MobiCom'99, Washingtion, USA, 1999, pp. 263-270.

[11] Akyildiz, I. F., W. Su, Y. Sankarasubramaniam, and E. Cayirci. 2002. Wireless sensor networks: a survey. Computer Networks 38 (4): $393-$

422. http://www.sciencedirect.com/science/article/B6VRG44W46D4-1/2/f18cba34a1b0407e24e97fa7918cdfdc

[12] Romer, K. and F. Mattern (2004). The design space of wirelesssensor networks. Wireless Communications, IEEE [see also IEEE Personal Communications] 11 (6), 54-61. 
[13] ZigBee Alliance, “ZigBee PRO Specification", Standard, Oct. 2007.

[14] HART Communication Foundation, "HART Field Communication Protocol Specification, Revision 7.0", Standard, Sept. 2007.

[15] ISA100 Standards Committee, "ISA100.11a-2009 Wireless systems for industrial automation: Process control and related applications", Standard, Sept. 2009.

[16] Stig, P., C. Simon, and S. Amund. 2008. Layered Software Challenge of Wireless Technology in the Oil $\backslash \&$ Gas Industry. In Proceedings of the 19th Australian Conference on Software Engineering. IEEE Computer Society.

[17] Craig, William C .Working with Wireless Control . 2006.

In Tech, v53 n1 p49 (1).

[18] Akyildiz, I.F., et al.,"A Survey of Sensor Networks", IEEE Communications Magazine,

[19] A. Talevski, S. Carlsen2, and S. Petersen, Intelligent Wireless Methodologies and Technologies

[20] Pottie, G. J. 1998. Information Theory Workshop, 1998, Wireless sensor networks.

[21] Carlsen, S., Petersen, S., Skavhaug, A. and Doyle, P., "Using Wireless Sensor Networks to Enable Increased Oil Recovery", Proceedings of the 13th IEEE International Conference on Emerging Technologies and Factory Automation, Hamburg, Germany, Sept. 15-18, 2008, pp. 1039-1048.

[22] Petersen, S., et al., "A Survey of Wireless Technology for the Oil \& Gas Industry", Proceedings of the SPE Intelligent Energy Conference, 25-27 Feb. 2008.

[23] Ferreira, V.H, Alves da Silva, A.P, "Toward Estimating Autonomous Neural Network-Based Electric Load Forecasters", IEEE Transactions on Power Systems, Volume 22, Issue 4, November 2007, pp: 1554-1562

[24] Energy Model for H2S Monitoring Wireless Sensor Network Chao Xiaojuan, Waltenegus Dargie and Lin Guan

[25] R.J.Reiffenstein, W.C.Hulbert, and S.H.Roth. Toxicology of hydrogen sulphide. Annu. Rev. Pharmacol. Toxicol, 32(1):109-134, 1992.

[26]Todorovski, M., and D. Rajicic. 2006. An procedure in solving optimal power flow by IEEE initialization genetic algorithm. Power Systems, Transactions on 21 (2): 480-487.

[27] Well Remote Monitoring System (cited 200918 January); Available from:

http://www.evoc.com/oversea/solutions/Well-Remote Monitoring-System.aspx
[28] Martin, D., E. Erik, V. Aart Joakim in't, G. Stein, L. Tor Sverre, H, R. vard Kolle, S. Oddvar, r, and sen. 2008. Wireless Sensor Networks for Off-shore Oil and Gas Installations. In Proceedings of the 2008 Second International Conference on Sensor Technologies and Applications. IEEE Computer Society. 